\title{
Eyelid myoclonia with typical absences: an epilepsy syndrome
}

\author{
R E Appleton, C P Panayiotopoulos, B A Acomb, M Beirne
}

\author{
Department of \\ Neurology and \\ Neurophysiology, \\ Royal Liverpool \\ Children's NHS Trust \\ (Alder Hey), \\ Liverpool, UK \\ R E Appleton \\ B A Acomb \\ M Beirne \\ Department \\ of Clinical \\ Neurophysiology and \\ Epilepsy, St Thomas' \\ Hospital, London, UK \\ C P Panayiotopoulos \\ Correspondence to: \\ Dr R E Appleton, \\ Department of Neurology \\ and Neurophysiology, Royal \\ Liverpool Children's NHS \\ Trust (Alder Hey), Eaton \\ Road, Liverpool L12 $2 \mathrm{AP}$. \\ Received 26 May 1992 \\ and in revised form \\ 11 January 1993. \\ Accepted 1 April 1993
}

Abstract

Five unrelated patients are described with the clinical and electrical features of eyelid myoclonia with absences (EMA). In this syndrome brief, typical absences occur with rapid eyelid myoclonia associated with retropulsive movements of the eyeballs and occasionally of the head. The seizures are of shorter duration than in childhood absence epilepsy, and are accompanied by less profound impairment of consciousness. The electroencephalogram demonstrates high amplitude discharges consisting of spikes, multiple spikes and slow waves at a fluctuating frequency of 3-5 $\mathrm{Hz}$ and following eye closure, which disappear in darkness. Photosensitivity is also seen. Onset is in early childhood and EMA appears to persist into adult life. Treatment is sodium valproate in combination with either ethosuximide or a benzodiazepine. On the basis of the clinical features, EEG findings, and the

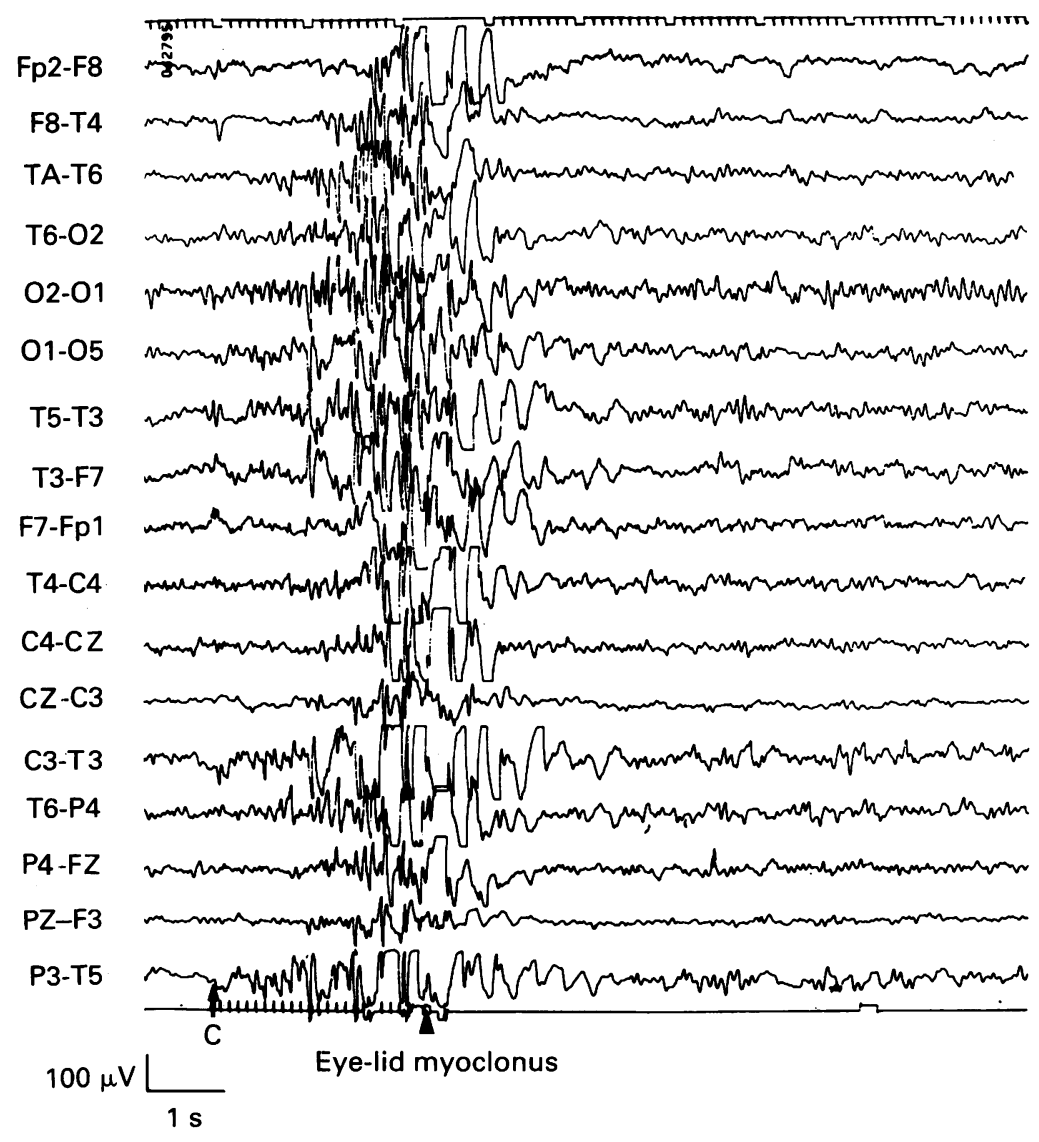

Figure 1 EEG recording; response to photic stimulation at 10-12 Hz with eyes closed (C)-high amplitude spike and wave and polyspike discharges with eyelid myoclonia (EM) and momentary, impaired consciousness. response to treatment and prognosis, it is suggested that EMA be classified as a specific epilepsy syndrome.

\section{(F Neurol Neurosurg Psychiatry 1993;56:1312-1316)}

The Commission on the Classification and Terminology of the International League Against Epilepsy ${ }^{1}$ has provided a syndromic and disease classification of epilepsies. A specific epilepsy syndrome is defined on the basis of the age of onset of seizures, type(s) of seizures, family history, neurological examination, and findings on the ictal and interictal EEG. The purpose of this paper is to propose that eyelid myoclonia with absences (EMA), first described by Jeavons ${ }^{23}$ as a form of photosensitive epilepsy, has distinct clinical and EEG features which justify its recognition as a separate epileptic syndrome and should be included in the future classifications of the epilepsies.

\section{Patient 1}

This girl presented at the age of 2.5 years with repeated episodes of looking up, eyelid jerks, and brief lapses in concentration. The child's parents thought that these episodes were caused by the wind on her face as they usually occurred on leaving a building and going outside. The patient's birth, perinatal history, and early development had been normal. There was no family history of epilepsy and no parental consanguinity. At the age of 5 years the patient was referred to the School Medical Officer by her teachers because of an increasing frequency of these episodes, which seemed to distress her. Neurological examination was normal. A diagnosis of a habit mannerism or tic was made. The episodes persisted but her development and academic performance remained normal. At the age of 7 years, while watching television from approximately half a metre, she was found on the floor, pale, conscious but confused. Neurological and physical examinations were normal. EEG demonstrated a normal background with eyes open. Multiple spikes and spike-slow wave complexes were seen on eye closure which were associated with occasional, brief, upward movements of the eyelids and very brief absences (1-3 s) which attenuated on eye opening and also in darkness. Photic stimulation evoked numerous, generalised, high amplitude discharges of irregular polyspike and slow wave activity at a flash frequency of $8-12 \mathrm{~Hz}$ (fig 1). Carbamazepine had no effect on the seizures. 
Figure 2 Video-EEG showing high amplitude polyspike and spike-wave activity on eye closure, and associated with eyelid and head myoclonia. ( $C=$ on closing eyes; $O=$ on opening eyes).
Figure 3 Video-EEG demonstrating normal background activity when eyes open $(O)$, but on eye closure $(C)$, frequent, generalised, high amplitude, irregular polyspike and spike-wave discharges associated with eyelid myoclonus (EM).

Fp2-F4

F4-C4

C4-P4

$\mathrm{P} 4-\mathrm{O} 2$

Fp1-F3

F3-C3

C3-P3

P3-01

Fp2-F8

F8-T4

T4-T6

T6-O2

Fp1-F7

F7-T3

T3-T5

T5-01

$100 \mu \mathrm{V}$

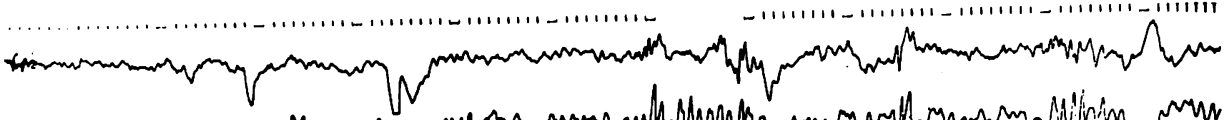

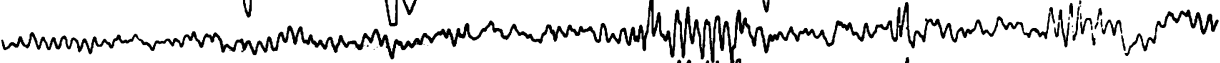



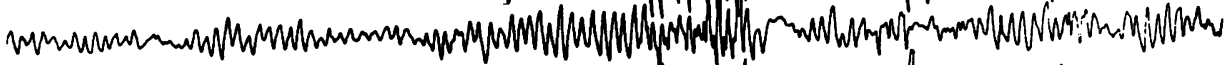

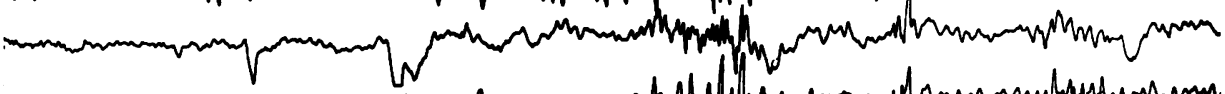

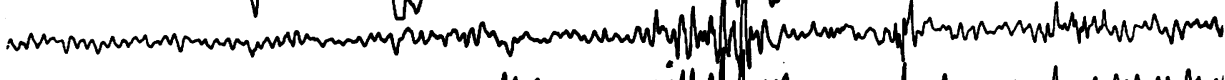

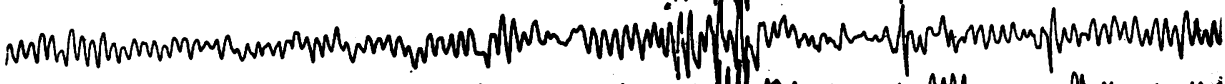

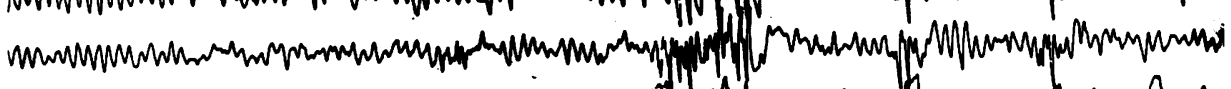

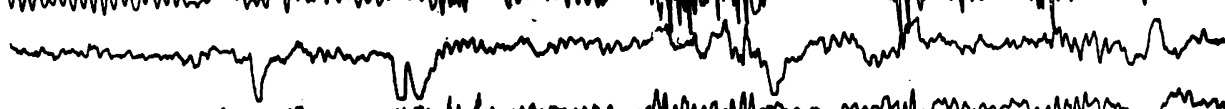

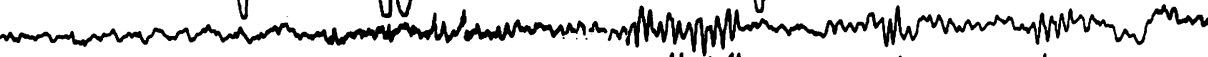

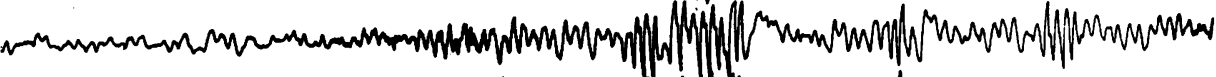

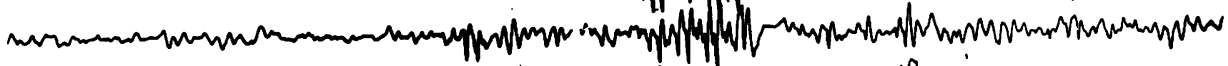
(n)

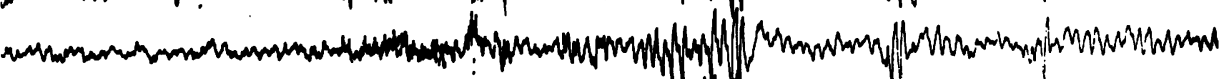

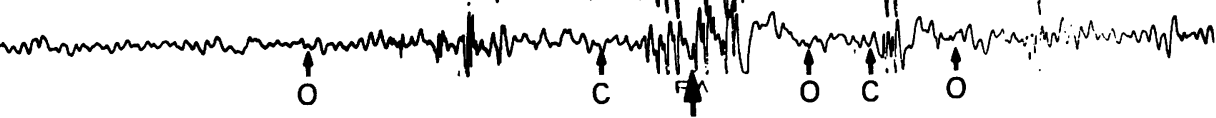

Eye-lid myoclonus

$1 \mathrm{~s}$

Sodium valproate (up to a maximum of $20 \mathrm{mg} / \mathrm{kg}$ per day) reduced the frequency of the seizures. Her parents were initially reluctant to continue treatment in an increased dosage, because of increased appetite and weight gain. The addition of ethosuximide $(20 \mathrm{mg} / \mathrm{kg}$ per day) to valproate has virtually
Fp2-F4

F4-C4

C4-P4

$\mathrm{P} 4-\mathrm{O} 2$

Fp1-F3

F3-C3

C3-P3

P3-01

Fp2-F8

F8-T4

T4-T6

$\mathrm{T} 6-\mathrm{O} 2$

Fp1-F7

F7-T3

T3-T5

T5-01

$100 \mu \mathrm{V}$

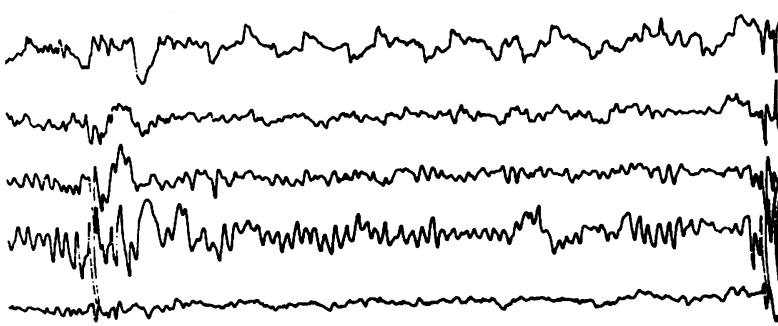

(1)

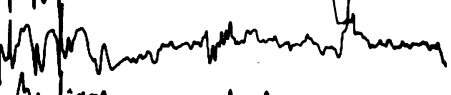

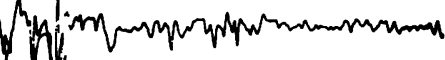

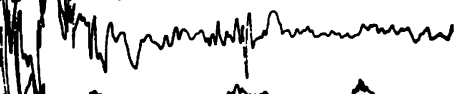

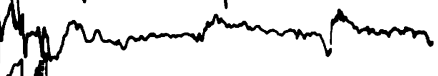

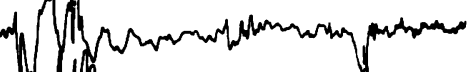

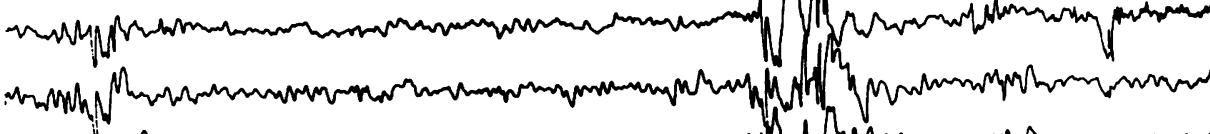

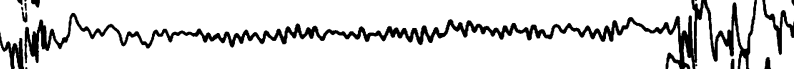

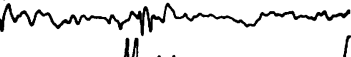

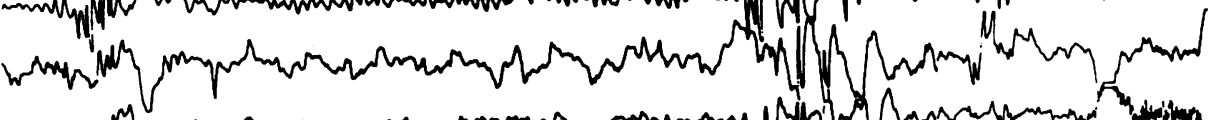

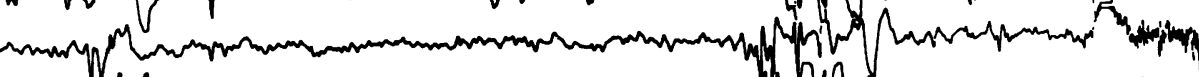

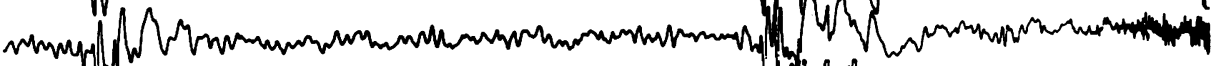

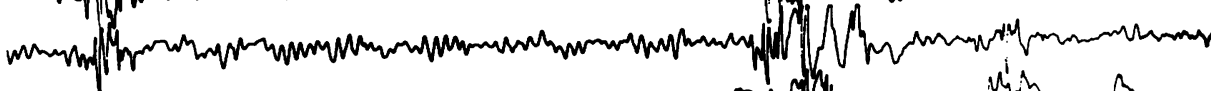

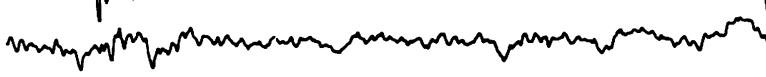

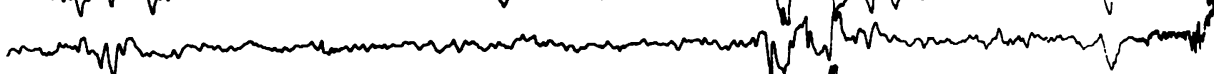

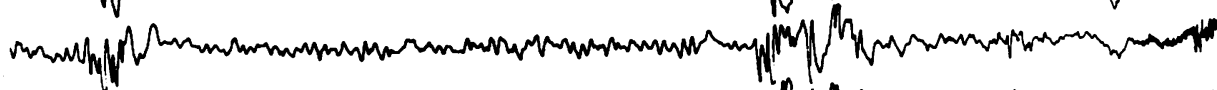

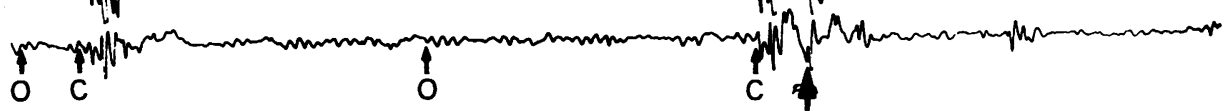

Eye-lid myoclonus 

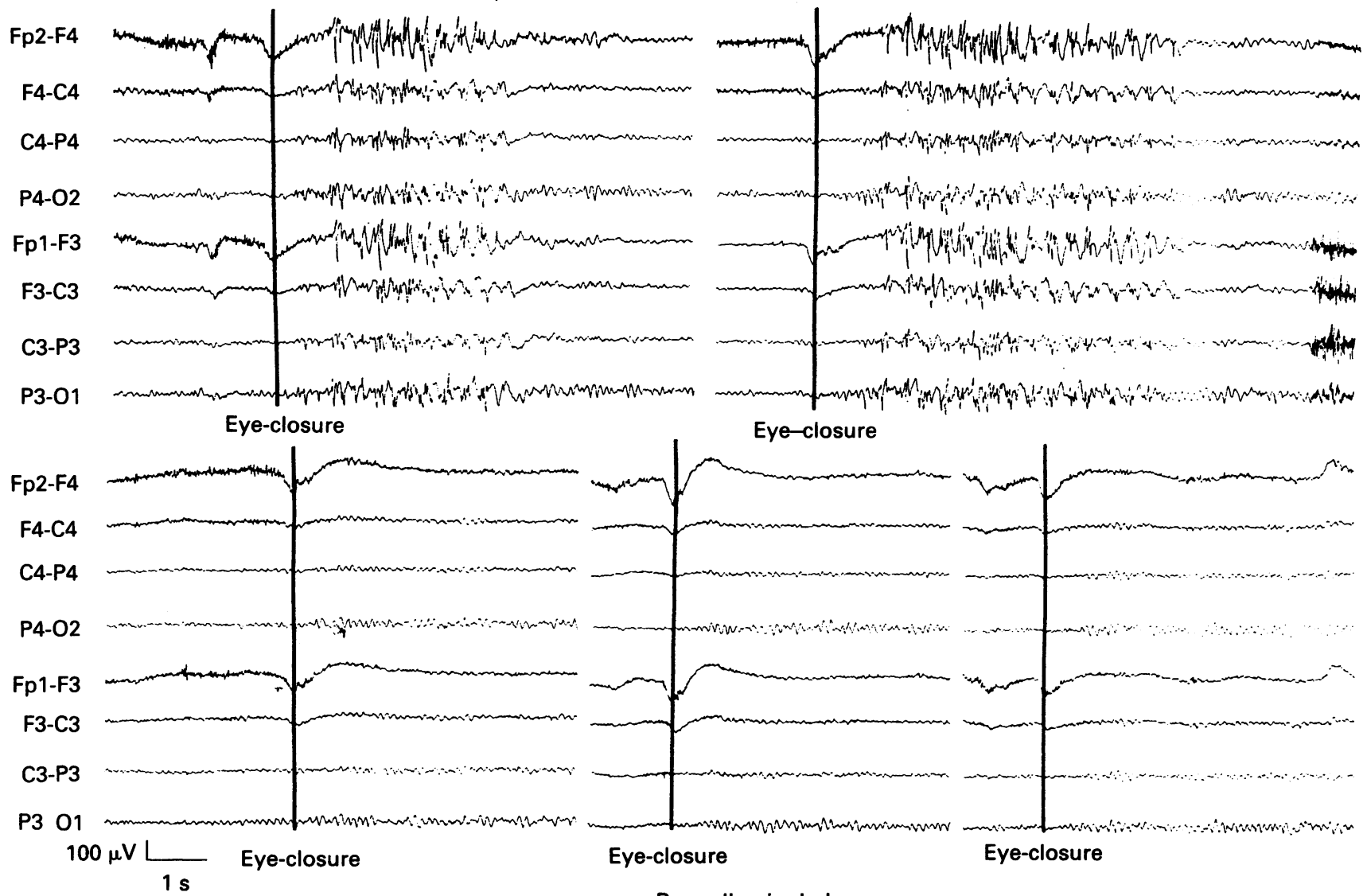

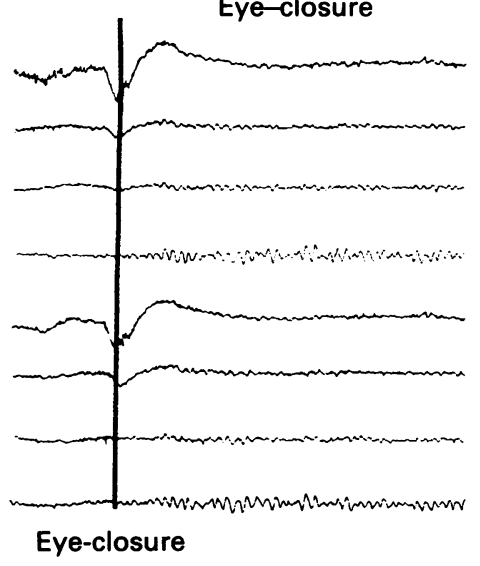

Recording in darkness

Figure 4 Video-EEG recording at 33 years of age; Upper traces: EEG showing high amplitude spikes and multiple spikes and slow waves occurring on eye closure in normal light. The discharges were frequently associated with eyelid myoclonia and a very brief, mild impairment of consciousness. Lower traces: total darkness inhibited the abnormalities that were induced by eye closure.

with a 4-month history of eyelid jerking and 'blanks'. Consciousness was not lost. The episodes could occur up to a maximum frequency of 10 per hour and were more frequent when the patient was tired; when this occurred, the patient appeared upset and would run to his mother or father. The patient's birth, perinatal history and early development had been normal. There was no family history of epilepsy and no consanguinity. Neurological examination was normal. A diagnosis of a simple motor tic was made by the family doctor. At 4 years of age, an EEG was undertaken, due to the persistence of the episodes. This demonstrated normal activity when the eyes were open but on eye closure, there were frequent generalised spike-wave and polyspike discharges, accompanied by upward jerks of the eyelids (fig 2), which did not occur in the dark. Photic stimulation produced generalised raised amplitude, irregular polyspike and slow wave discharges. Hyperventilation induced a very brief $(3 \mathrm{~s})$ clinical absence with generalised $3 \mathrm{~Hz}$ spike and slow wave activity. Sodium valproate (in a dosage of $15 \mathrm{mg} / \mathrm{kg}$ per day), carbamazepine, phenobarbitone, and phenytoin had no obvious effect. Nitrazepam resulted in an increase in seizure frequency and intolerable irritability. Ethosuximide produced some improvement. At the age of 8 years the patient became confused and agitated and subsequently vacant while working at a computer. Sodium valproate $(30 \mathrm{mg} / \mathrm{kg}$ per day) was added to ethosuximide, following which there was a dramatic reduction in seizure frequency. The patient is now aged 13 years and continues to experience very brief episodes of eyelid myoclonia with a downward tilt of his head and an absence when emerging into bright light. His academic performance remains in the above average range.

\section{Patient 3}

This girl was seen by a paediatric neurologist at the age of 4.5 years with a 4-month history of frequent episodes characterised by brief, upward jerking movements of her eyes and eyelids and trances. These episodes had only been observed while the patient was watching television or having emerged into bright sunlight. The patient's birth, perinatal history and early development had been unremarkable. There was no family history of epilepsy. Neurological examination was normal, EEG demonstrated normal background activity when the eyes were open but on eye closure there were generalised, high amplitude, irregular polyspike and spike-wave discharges associated with upward jerks of the eyelids and head and a brief absence (fig 3). Photic stimulation produced persistent and generalised, high amplitude polyspike and slow 
Figure 5 Video-EEG recording at 31 years of age; high amplitude discharges of typical spike and slow wave occurring mainly on eye closure, and associated with rapid eyelid myoclonus and upward deviation of the eyeballs, and mild impairment of consciousness.

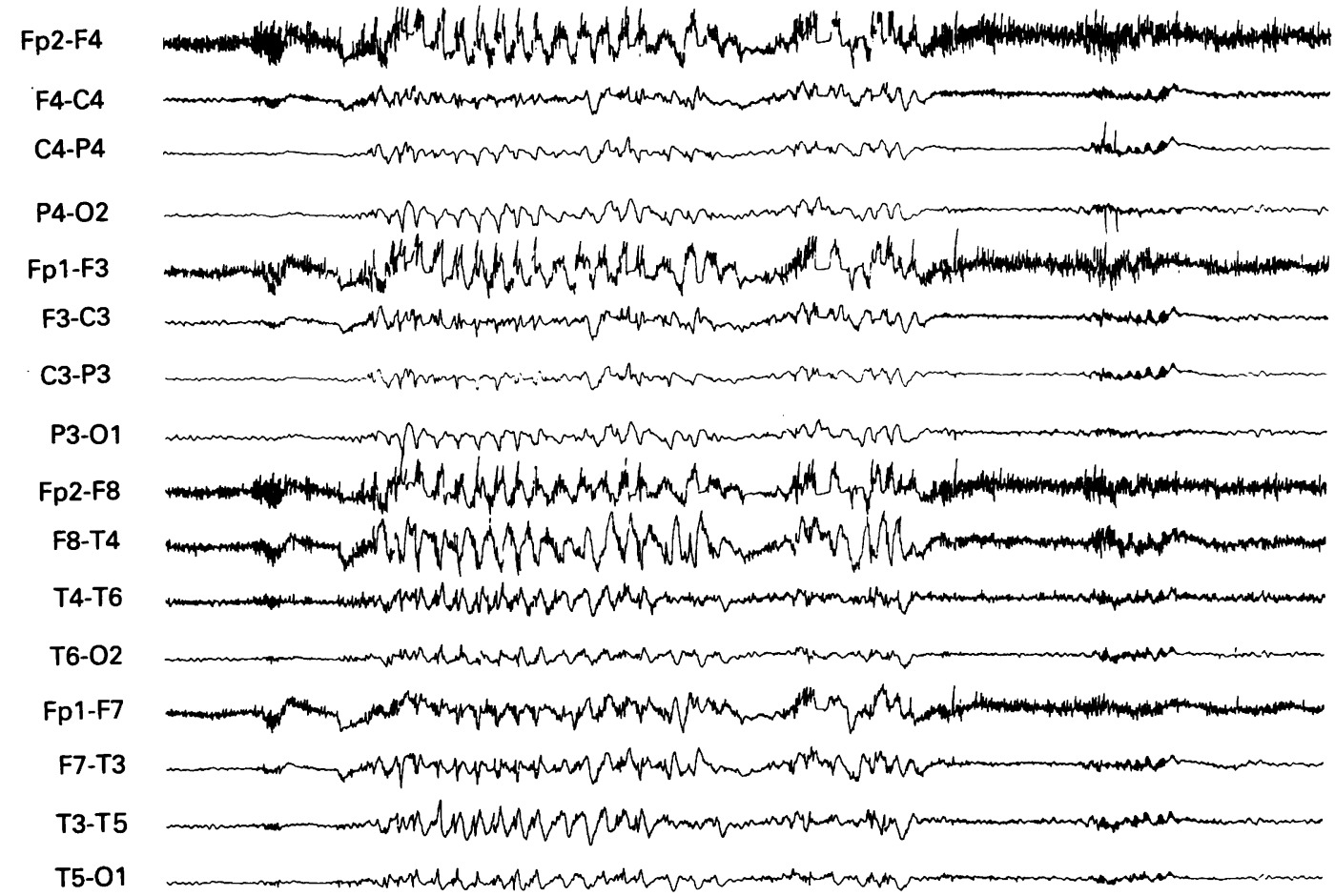

wave activity at all flash frequencies. Hyperventilation accentuated this and also produced a brief and generalised $3 \mathrm{~Hz}$ spike and slow wave activity accompanied by a clinical absence with a brief, backward jerk of the head. Sodium valproate $(40 \mathrm{mg} / \mathrm{kg}$ per day) abolished the absences and has reduced the eyelid myoclonia. Her early academic performance has been normal.

\section{Patient 4}

A 17-year-old girl was referred having had two generalised tonic-clonic seizures one of which had occurred while travelling in a car with the sun flickering on the road through the trees. The patient also complained of brief episodes during which she experienced 'misting' in front of her eyes, a lapse in concentration and restlessness. Retrospectively, the patient's mother had noted episodes which had occurred from three years of age, when the girl's eyelids would jerk rapidly for 2-3 s with associated retropulsive movements of the eyeballs; these episodes were still occurring many times a day, but the patient was unaware of them. Between the ages of 22 and 33 years the patient experienced almost monthly generalised tonic-clonic seizures, most of which occurred soon after waking. Academic performance at school has been average. There was no relevant family or past medical history. Neurological examination was normal. EEG revealed generalised spike and wave discharges (fig 4) and photosensitivity. Treatment with sodium valproate alone, and in combination with phenytoin, was of no benefit. Sodium valproate with primidone $(250 \mathrm{mg}$ at night) resulted in resolution of the tonic-clonic seizures. The addi- tion of ethosuximide to sodium valproate and primidone was followed by an over $75 \%$ reduction in the EMA seizures. The patient is now aged 35 years, married, and an accountant.

\section{Patient 5}

This girl developed daily, typical absences at 4.5 years of age. Most lasted less than $10 \mathrm{~s}$ and were associated with jerks of the eyelids and upward rotation of the eyeballs. The patient's past medical history was unremarkable and there was no family history of epilepsy. Neurological examination was normal; EEG was not performed. Between the ages of 6 and 30 years the patient had six generalised tonic-clonic seizures. At 31 years of age EEG performed after partial sleep deprivation revealed the following: within seconds of awakening, there were frequent, generalised discharges of spike and multiple spike and slow waves at 3-4 Hz, lasting for between 3 and $5 \mathrm{~s}$, but occasionally for up to 7-10 s (fig 5). During the discharges the patient opened her eyes with eyelid and eye brow myoclonus. There was also a slight backward jerk of the head, and an occasional jerk of the eyeballs. The patient stopped counting during the discharges. Most of the discharges occurred during eye closure but they also occurred when the eyes were closed but not in the dark. There was marked photosensitivity. The patient has never experienced any limb myoclonus. Treatment with ethosuximide reduced but did not abolish the absences; the addition of carbamazepine was associated with a deterioration. The patient has become virtually seizure-free on a combination of ethosuximide and sodium valproate. 
The patient is currently aged 32 years, married, and a successful sales manager.

\section{Discussion}

Eyelid flickering or fluttering, which may resemble spontaneous eye-blinking, may occur in childhood and juvenile absence epilepsy, ${ }^{14}$ symptomatic absence epilepsy, ${ }^{5}$ fixation-off sensitive epilepsy, ${ }^{6}$ and benign myoclonic epilepsy of infancy. ${ }^{7}$ Eyelid flickering or fluttering is distinct, and must be differentiated from eyelid myoclonia which occurs simultaneously with spike-wave or polyspike discharges, ${ }^{38}$ and is the hallmark of EMA.

EMA appears to be rare; whether this is real or reflects underdiagnosis (or underreporting) is unclear. It is characterised clinically by myoclonic jerks of the eyelids with upward deviation of the eyes (and occasionally the head), associated with very brief absences and, on EEG, by generalised spike-wave or polyspike-wave discharges which occur on eye closure and also following intermittent photic stimulation. At other times, clinical absences may occur both spontaneously on awakening and also on hyperventilation. Limb myoclonus has been described rarely, ${ }^{8}$ but was not seen in our patients. Tonic-clonic seizures may occasionally develop but are infrequent, and occur usually after precipitating factors such as sleep deprivation or photic stimulation. Most patients are photosensitive, frequently showing a photoconvulsive response. The age of onset of the disorder is similar to that of typical absence epilepsy (between the ages of 3 and 7 years) but unlike the photosensitive epilepsies which tend to develop around puberty. Seizure control in EMA is usually more difficult than in absence and other generalised epilepsies, ${ }^{89}$ and it is thought that remission before adulthood is less likely. Sodium valproate with either ethosuximide or, occasionally clobazam, appears to be more effective in treating this condition than if a single drug is used alone. There is relatively little else known about this type of epilepsy, and specifically the natural history and effect on cognitive function.

Our patients demonstrate the characteristic clinical and EEG features of EMA, including the age of onset and response to treatment. Two of the five patients were diagnosed initially as having a tic or mannerism with, in patient 1 , a subsequent delay in diagnosis of almost eight years with no obvious detrimental effect on development or academic progress. This suggests that this epilepsy may be relatively benign although it is possible that continued, untreated absences could result in some subtle cognitive dysfunction, as has been reported in adults who have had untreated absences throughout childhood. ${ }^{10}$ Only patient 4 experienced frequent gener- alised tonic-clonic seizures, and this probably reflected initially inappropriate anti-epileptic medication. Although there is very little information on the long term prognosis in EMA, there is clear evidence (patients 4 and 5 and also a personal communication of Dr JBP Stephenson, Glasgow) that the condition persists into at least the third decade with no obvious detrimental effect on intellectual function.

Binnie and Jeavons ${ }^{3}$ consider EMA to be a type of photosensitive epilepsy, but make the important point that EMA must be differentiated from self-induced seizures. ${ }^{311}$ Our patients did not admit to self-inducing the seizures over many years of follow-up (either spontaneously or on direct questioning) and self-induction was not demonstrated following repeated and prolonged video-EEG recordings. The eyelid myoclonia never preceded the epileptiform discharges and the abnormal electrical discharges were not seen in the dark-for example, fig 4, patient 4 . Finally, the patients were of average or above average intelligence with no psychosocial or psychiatric problems, in contrast to a previous report, ${ }^{11}$ and all our patients expressed obvious relief when seizure control was achieved.

This syndrome has not hitherto been recognised by the International League Against Epilepsy. Our observations and those of others, ${ }^{389}$ indicate that EMA should be classified as a specific epilepsy syndrome within the idiopathic generalised epilepsies.

The authors are grateful to Mrs Linda Finnegan for her patience and assistance in the preparation of this manuscript.

1 Commission on Classification and Terminology of the International League Against Epilepsy. Proposal for classification of epilepsies and epileptic syndromes. Epilepsia 1989;30:389-99.

2 Jeavons PM Nosological problems of myoclonic epilepsies in childhood and adolescence. Dev Med Child Neurol 1977;19:3-8.

3 Binnie CD. Jeavons PM. "Photosensitive epilepsies" in epileptic syndromes of infancy, childhood and adolescence, 2nd edn. Roger J, Bureau M, Dravet C, Dreifuss FE, Perret A, Wolf P, eds, London: John Libbey Eurotext, Perret A, Wolf

4 Panaviotopoulos CP, Obeid T, Waheed G. Differentiation of typical absence seizures in epileptic syndromes. Brain 1989;112:1039-56.

5 Panayiotopoulos CP, Chroni E, Daskalopoulos C, Baker A, Rowlinson S, Walsh P. Typical absence seizures in adults: clinical, EEG, video-EEG findings and diagnostic/syndromic considerations. $₹$ Neurol Neurosurg Psychiatry 1992;55:1002-8.

6 Panayiotopoulos CP. Fixation-off-sensitive epilepsy in eyelid myoclonia with absence seizures. Ann Neurol 1987;22:87-9.

7 Dravet $\mathrm{C}$, Bureau M, L'epilepsie myoclonique benigne du nourrison. Rev Electroencephalogr Neurophysiol Clin nourrison. Rev

8 De Marco P. Eyelid myoclonia with absences (EMA) in two monovular twins. Clin Electroencephalogr 1989;20: two m.

9 Gobbi $\dot{M}$, et al. Le mioclonie palpebrali con assenza alla chiusura degli occhi. Boll Lega It Epil 1985;51/52:225-6.

10 Lousieau P, Pestre M, Dartigues J F, Commenges D Barbeger-Gateau C, Cohado S. Long-term prognosis in two forms of childhood epilepsy: typical absence seizures and epilepsy with rolandic (centro-temporal) EEG foci. Ann Neurol 1983;13:642-8.

11 Binnie CD, Darby CE, de Korte RA, Wilkins AJ. Selfinduction of epileptic seizures by eyeclosure: incidence and recognition. 7 Neurol Neurosurg Psychiatry 1980; 43:386-9. 